\title{
Importancia del curriculum oculto en el proceso de enseñanza - aprendizaje
}

\author{
Prof. Dra. Gladys Brítez ${ }^{1}$ \\ gladysbritez200@yahoo.com.ar \\ Universidad Nacional de Pilar
}

\section{RESUMEN}

En busca del potenciamiento de la Educación, a través de fortalecimiento curricular que permitan ofrecer una formación académica contemporánea e idónea para estos tiempos respecto a la articulación con la sociedad, se aborda el tema del Curriculum Oculto y su importancia en el proceso de enseñanza - aprendizaje, partiendo del reconocimiento de la presencia del mismo y la actuación del educador y educando en su aplicación. Se trata de una investigación bibliográfica basada en datos secundarios obtenido a través de diversas fuente preseleccionadas y relacionadas entre sí. Se pretende determinar la importancia del Curriculum Oculto y para ello se describen sus características y aplicación en el proceso de enseñanza - aprendizaje. Finalmente se resalta el potencial con el que cuenta el Curriculum Oculto para influir en dicho proceso y la necesidad de direccionar dicha influencia para el complemento al Curriculum Formal.

Palabras clave: curriculum oculto; curriculum formal; enseñanza - aprendizaje.

\footnotetext{
${ }^{1}$ Doctora en Gestión Educacional por la Universidad Nacional de Itapúa -Magister en Ciencias de la Educación por la Universidad Nacional de Pilar - Especialista en Psicopedagoga, Metodología de la Investigación y Dedicativa Universitaria por la Universidad Nacional de Pilar - Catedrática de Metodología de la Investigación y otros
} 


\title{
Importance of the hidden curriculum in the teaching - learning process
}

\begin{abstract}
In search of the empowerment of Education, through the curricular strengthening that allows to offer a contemporary and adequate academic training for these times with respect to the articulation with society, the subject of the Hidden Curriculum and its importance in the learning process is addressed. - Learn, starting from the recognition of the presence of the same and the action of the educator and educating in its application. It is a bibliographic research based on secondary data obtained through different preselected and related sources. If you want to determine the importance of the Hidden Curriculum and therefore if you describe its characteristics and application in the learning process - learning. Finally, it highlights the potential with which it handles the Hidden Curriculum to influence the process and the need to guide this influence to complement the Formal Curriculum.
\end{abstract}

Keywords: hidden curriculum; formal curriculum; teaching - learning.

Artículo recibido: 02 noviembre. 2021 Aceptado para publicación: 28 noviembre 2021 Correspondencia: gladysbritez200@yahoo.com.ar Conflictos de Interés: Ninguna que declarar 


\section{INTRODUCCIÓN}

Según (Ruíz, 2020) la educación es acción de intervención, es el proceso de enseñanza enfocado al aprendizaje de múltiples contenidos, en dicha acción y proceso se pretende dotar al educando de capacidades para sobrevivir dentro de una cultura o integrarlo en la reducción de necesidades y del largo de las aspiraciones de la comunidad, así como trasmitirle las capacidades de tener conocimiento de su presente a la luz de su pasado histórico social y eventualmente poder transformarlo.

Es tan basto en contenido a enseñar que no alcanza a ser impartido en su totalidad por lo que se prioriza los fundamentar según el tiempo y lugar, al mencionar tiempo no solamente se habla del lapso medido en horas de enseñanza y aprendizaje; sino que se hace referencia al tiempo en la historia mundial y local donde la educación es ejecutada, lo mismo se dice del lugar, no solo el lugar físico si no la posición o roll que ejerce cada individua en la historia de la humanidad.

La palabra currículo tiene muy diversos significados, por lo que se considera un término polisémico, su acepción dependerá de la concepción, teoría y modelo que lo sustente así como del enfoque pedagógico que lo circunscriba, en relación a las condiciones sociales, políticas y económicas en las cuales se desarrollan los sistemas educativos, los que reflejan las relaciones de poder político y de control de los sistemas hegemónicos, que históricamente han generado las tensiones y problemáticas que la educación las debe atender. (Toro Santacruz, 2017) Así mismo, la noción de currículo además de polisémica es polimorfa, se encuentran múltiples definiciones, diferentes corrientes, posturas teóricas y variadas interpretaciones. Se detectan diversos significados que a lo largo de la vida ha tenido el concepto de currículo, así como inercias y vacíos en los procesos institucionales y la búsqueda del horizonte de sentido en el que podríamos comprometernos para la reorientación de la vida académica y de nuestra práctica pedagógica en el ámbito de la formación universitaria (Del Basto, 2005).

El concepto de currículum aparece muy tardíamente en la producción del pensamiento educativo, en las publicaciones, así como su uso entre el profesorado. El Diccionario de la RAE no lo recoge hasta su edición de 1983, distinguiendo una doble acepción: de plan de estudio (proyecto a ser recorrido) y de currículum vitae (proyecto ya pasado). En ese mismo 
año, en el registro de publicaciones con ISBN español una única publicación llevaba en su título alguno de los términos: curriculum o currículum. (Sacristan J. G., 2010).

Partiendo de la premisa de la necesidad de enseñar contendidos prioritarios, se introduce el tema del curriculum, por un lado, un curriculum formal y por otro el curriculum complementario, este último es el objeto de estudio de este trabajo. Se pretende determinar la importancia del curriculum oculto y para ello se describen sus características y aplicación en el proceso de enseñanza - aprendizaje.

\section{MATERIALES Y MÉTODOS}

El estudio es una investigación bibliográfica seleccionado entre los tipos de investigaciones posibles por la posibilidad de cubrir una amplia gama de fenómenos ya que abarca una realidad espacio-temporal mucho más dilatada en comparación a estudios que requieren delimitaciones temporales más ajustadas (Sampieri, Fernández, Baptista, 1991).

En cuanto a la recolección de datos, como fuente de información fueron utilizados datos secundarios entre los que se encuentran materiales bibliográficos citados a lo largo del trabajo y cuyas referencias son comprobables al término del mismo. Para la elección de cada material fue necesaria la búsqueda a través de internet basada en las palabras claves de la investigación como: curriculum, educación, enseñanza, aprendizaje. Posteriormente se procedió al relacionando datos existentes de distintas fuentes lo que proporciono una visión panorámica y sistemática del tema abordado con la combinación de múltiples fuentes dispersas.

Sobre el análisis de datos posterior a la búsqueda y recopilación de los mismos, se requirió una organización, valoración, crítica e información de datos bibliográficos. Esto se realizó teniendo en cuenta la relevancia de las fuentes y el contenido de la misma donde fueron desechadas las incoherencias o contradicciones.

\section{RESULTADOS Y DISCUSIÓN}

\subsection{Concepción del Curriculum Oculto}

En términos educativos, el currículum corresponde a los planes y programas de estudio, o el conjunto de contenidos, en un sentido amplio, que, organizados en una determinada secuencia, el sistema educativo se compromete a comunicar. Al mismo tiempo, el currículum es una selección cultural con propósitos formativos, que organiza la trayectoria del educando 
en el tiempo y que, en los contenidos, esquemas mentales, habilidades y valores que contribuye a comunicar, es un regulador mayor de su experiencia futura (Cazares, 2013).

El objetivo principal de un currículo es plasmar una concepción educativa, misma que constituye el marco teleológico de su operatividad. Por ello, para hablar del currículo hay que partir de qué se entiende por educación; precisar cuáles son sus condiciones sociales, culturales, económicas, etc. Su real función es hacer posible que los educandos desenvuelvan las capacidades que como personas tienen, se relacionen adecuadamente con el medio social e incorporen la cultura de su época y de su pueblo (Guzman Paz, Teoría curricular, 2012).

Pansza, M., mencionado por (Pérez Pérez, 2014) propone que el currículo representa una serie estructurada de experiencias de aprendizaje que en forma intencional son articuladas con una finalidad concreta que es: producir los aprendizajes esperados. Para lograr esto se tienen dos aspectos interconectados: el diseño y la acción. Implican una concepción de la realidad, del conocimiento y del aprendizaje.

Para (Murillo Pacheco, 2010) el currículum se considera como el proyecto que preside las actividades educativas escolares, precisa sus intenciones y proporciona guías de acción adecuadas y útiles para los profesores que son responsables directos de su puesta en marcha (Coll, 1992:31). El proyecto conjuga la teoría y la práctica, el deber ser y el ser. Lo constituyen los docentes, las instalaciones educativas, la sociedad, los padres, los alumnos, las instituciones gubernamentales y privadas, es decir, toda instancia aplicada en su elaboración y desarrollo. En términos generales comprende los siguientes aspectos:

- Fundamentación

- Identificación de la carrera

- Perfil de ingreso y egreso

- Estructura y organización curricular

- Programas de las materias, áreas o módulo

- Y otros

El currículo puede concebirse de diferentes formas: como producto, es decir como el documento resultado de la planeación y, en este sentido sería un concepto limitado al plan de estudios y su organización de asignaturas; como proceso en el que además del plan de estudios y su estructura académica implica el proceso que genera su puesta en marcha y como 
práctica social, o sea el proyecto curricular inserto en un contexto y realidad social que se vive como una práctica social de sujetos reales (Alonso Tejeda, 2009).

(Cazares, 2013) expone algunas definiciones ordenadas cronológicamente que en alguna medida tratan de definir el currículum:

"Básicamente el currículum es lo que ocurre a los niños en la escuela como consecuencia de la actuación de los profesores. Incluye todas las experiencias de los niños por las que la escuela debe aceptar responsabilidades" (Tyler 1949:34).

"Una serie de unidades de contenidos organizadas de tal manera que el aprendizaje de cada unidad pueda ser logrado por un acto simple apoyado por las capacidades especificadas de las unidades anteriores (en la secuencia) y que ya han sido dominadas por el alumno" (Gagné, 1966:77).

"Currículum es una serie estructurada de objetivos del aprendizaje que se aspira lograr. El currículum prescribe (o por lo menos anticipa) los resultados de la instrucción" (Johnson, 1967:28).

El Currículum Oculto, aunque no con esa misma denominación, era manejado ya desde hace varios años, y eso partido de la observación de los educandos donde se podía constatar que los mismo habían adquirido aprendizaje no contemplados en el Curriculum Formal compuesto por el Plan de Estudio y los materiales que respaldaban al mismo. (Durkeim, 1961).

Fue definido por (Jackson, 1968) como el conjunto de normas, costumbres, creencias, lenguajes y símbolos que se manifiestan en la estructura y el funcionamiento de una institución. Sin pretenderlo de manera reconocida, el Currículum Oculto constituye una fuente de aprendizajes para todas las personas que integran la organización. Los aprendizajes que se derivan del Currículum Oculto se realizan de manera osmótica, sin que se expliciten formalmente ni la intención ni el mecanismo o procedimiento cognitivo de apropiación de significados. 


\subsection{Características del Curriculum Oculto}

Puede considerarse que un currículo formal es como el marco explícito en el que se reproducen las relaciones culturales, económicas y sociales, mientras que el Currículo Oculto, refuerza y nutre las ideologías dominantes y los sistemas de creencias. Por lo que podría decirse que su característica más importante es que el mismo subyace a todas las experiencias educativas.

El escenario de análisis será siempre el aula, incluso si estuviéramos hablando de aulas virtuales o físicas, es preciso que en la investigación sobre curriculum que se desarrolla en las aulas, se utilicen metodologías más etnográficas y participantes, junto a marcos de análisis más amplios, en los que se tengan en cuenta las interrelaciones entre el sistema educativo y lo que acontece en otras esferas de la sociedad; de esta manera, es posible captar más fácilmente las conexiones entre el curriculum explícito y el curriculum oculto de la institución educativa y las producciones económicas (Rivera, 2015).

Otra característica del Curriculum Oculto es su influencia en los actores de la educación, (Vázquez, López y Calvo, 2019) explican que tradicionalmente el fracaso escolar y el abandono educativo han sido fenómenos cuyas causas son atribuidas al alumnado y su falta de interés hacia lo escolar, un nivel académico "de base" insuficiente, escasa habilidad para mantener la atención, etc. Sin embargo, el currículum, las prácticas docentes, el sistema educativo y su organización y sus exigencias no han formado parte del juicio sobre el fracaso y el abandono.

Desde una mirada holística e intersecciones, el currículum, el sistema educativo actual y la práctica docente deben ser cuestionadas como elementos que tienden a reproducir, pero también a producir las desigualdades y los dominios estructurales, disciplinarios e interpersonales que condicionan las vidas académicas y vitales del alumnado. Las personas que han fracasado en la escuela son aquellas que no responden a las expectativas de lo que el sistema entiende como alumnado ajustado al modelo esperado.

Así mismo resulta determinante respecto a la vinculación con lo educativo, las relaciones interpersonales que el alumnado mantiene con el profesorado, la confianza y las expectativas que este les tiene. La afectividad, el interés, la atención, la empatía, la confianza y las altas expectativas que el alumnado siente de su profesorado son directamente proporcional a su 
enganche escolar. El alumnado demanda reconocimiento a su persona, a sus logros, sus necesidades y dificultades; una atención personal que vaya más allá de lo estrictamente curricular y una mayor confianza en sus capacidades de superación. Son conscientes de que en secundaria las relaciones son diferentes, entendidas como débiles, jerárquicas, controladoras y curriculares, y basadas en el control y obtención de resultados académicos y no en el desarrollo de estrategias de desarrollo personal para afrontar las dificultades y presiones "extraescolares". Se pone en práctica el dominio interpersonal que incide en la construcción de identidades con baja autoestima (Vázquez, López y Calvo, 2019).

\subsection{Aplicación del Curriculum Oculto}

El Curriculum Oculto en aplicación es la inmersión del dialogo en el proceso de enseñanza - aprendizaje, en la interacción es considerando a cada actor educativo ya sea educando o educando, como una persona con ideas propias connadas a través de sus experiencias, experiencias que influye en sus opiniones y le permiten apreciar los temas planteados desde una perspectiva totalmente personal. No obstante, las interacciones que dan a cada actor la oportunidad de plantear sus ideas veden ser regidas por parámetros específicos, previamente acordados con todas las partes, para evitar varios focos de discusión que dispersen las ideas y no se haga posible la condensación del tema.

Es necesario hacer énfasis tomando las dificultades que se pueden llegar a tener en la relación dentro del aula lo dicho por Carrillo y Estrevel (2016), la construcción guiada del conocimiento y la comprensión, por tratarse de hechos sociales, se ve enmarcada y afectada a partir de los componentes de esta y, por tanto, lastrada con una carga cultural e ideológica que define, en concordancia con los valores imperantes, lo que va a ser considerado como buena enseñanza y un aprendizaje logrado, justificando lo que se va a aprender o no, cómo pensar, hablar, ver las cosas y valorar quién se expresa de acuerdo al habla deseada, sigue las reglas establecidas y prioriza lo que la comunidad escolar indica. Estas situaciones se pueden identificar en el aula, por parte de los docentes o bien por los estudiantes, no se trata de negar la opinión del otro, por el contrario, es cuestión de no anular y orientar al conocimiento que se desea que sea adquirido. Pero no se puede dejar de lado el conocimiento, sin las estrategias de enseñanza - aprendizaje que son una serie de pasos que se encamina a lograr un fin y las cuales van orientadas a la metodología utilizada por el 
docente y los propósitos de la educación. Estas variaran según los fines del curso, le metodología del centro de estudios, el contexto de la educación y la personalidad y preparación del docente. No se ahondará en las diversas estrategias de enseñanza pues sería tema de otro estudio (Aguilar, 2018).

\section{CONCLUSIÓN O CONSIDERACIONES FINALES}

Basados en los resultados obtenidos, se concluye sobre la importancia del Curriculum Oculto que: no basta solo con reconocer la existencia de un curriculum oculto, además en necesario analizar el contenido del mismo y la interacción que se produce en el proceso de enseñanzaaprendizaje, para así identificar la influencia del mismo en los educandos y hacer uso de mismo para cumplir con los objetivos de la educación a través de la vinculación de lo académico a lo social, cultural, ambiental y económico puesto que los aprendizajes en el aula están directa o indirectamente asociados con el desarrollo del educando y sus relaciones de dominación y subordinación en las áreas anteriormente citadas (Giroux, 1992).

A los educandos les resulta extremadamente difícil ir más allá de comprender su educación como una continuación del sistema que ha estado legitimando, impartiendo y evaluando su conocimiento durante sus años de formación. Ésta es una preparación más que inadecuada para el desarrollo humano y social, dado que evita muchos de los grandes desafíos, problemas y oportunidades reales, si no todos, asociados al aprendizaje y al crecimiento individuales y, concretamente, a un cambio y una transformación sociales más generales y positivos. Los estudiantes deben prestar más atención a la naturaleza de los problemas del mundo real y aprovechar las oportunidades de aprender una amplia gama de habilidades y capacidades procedentes de distintos campos de la experiencia y el conocimiento, y que les permiten enfrontarse a problemas complejos en diversos contextos.

Los retos educativos afrontados en este tiempo no solo requieren de una adecuación del Curriculum Formal, si no de la potenciación del Curriculum Oculto, éste puede ayudar a combatir desde el proceso de enseñanza-aprendizaje en las instituciones educativas y hasta articulaciones del sistema educativo con la sociedad como el caso de los problemas de inserción laboral, el espectro es amplio e incluso podría decirse que no tiene un límite de alcance indirecto ya que no solo apela a la memoria sino que es capaz de modelar patrones de conducta. 
Se propone a la institución educativa como campo propicio para la reflexión. Un clima positivo convoca a encuentros productivos; adicionalmente, el diálogo sincero y abierto se convierte en una plataforma de aprendizaje. Por consiguiente, el clima depende esencialmente de las actitudes de los integrantes de la comunidad educativa, pero también de la forma en que esa comunidad es dirigida por los administradores de la educación y de la valoración que de ella realice la sociedad.

Para el estudiante es necesario sentirse apoyado e instruido; lo cual genera un efecto de mejoramiento del desempeño, donde establece que el Curriculum Oculto incide en el rendimiento (Rivera, 2015).

\section{LISTA DE REFERENCIAS}

Aguilar, M. (2018). La influencia del Currículum Oculto en la formación de los estudiantes: Experiencia docente en las titulaciones de relaciones laborales y finanzas y contabilidad. Acciones e Investigaciones Sociales. issn: 1132-192X, Núm. 38 (2018). Págs. 99 a 118

Alonso Tejeda, M. E. (2009). Algunas consideraciones teóricas acerca del curriculum. Cuadernos para formación de profesores editado por la ENP. Obtenido de http://uiap.dgenp.unam.mx/apoyo_pedagogico/proforni/antologias/ALGUNAS\%20 CONSIDERACIONES\%20TEORICAS\%20ACERCA\%20DEL\%20CURRICULO. pdf

Casarini Ratto, M. (1999). Acercamiento al Currículum. México: Universidad Nacional Abierta. Dirección de Investigaciones y Postgrado.

Cazares, M. (2013). UNA REFLEXIÓN TEÓRICA DEL CURRÍCULUM Y LOS DIFERENTES ENFOQUES. (U. Cienfuegos, Ed.) Club de ensayos. Obtenido de https://www.clubensayos.com/Filosof\%C3\%ADa/UNA-REFLEXI\%C3\%93NTE\%C3\%93RICA-DEL-CURR\%C3\%8DCULUM-Y-LOS-

DIFERENTES/893948.html

Del Basto, L. M. (2005). Reflexión sobre el currículo universitario desde la teoría. ieRed: Revista Electrónica de la Red de Investigación Educativa (en línea) Vol 1, $N^{o} 3$. Durkheim, E. (1961). Moral Education. New York: Free Press 
García-de-León, B. A. (2020). Perspectiva radical del currículum oculto. Revista Guatemalteca de Educación Superior, 3(2), 58-68. Disponible en: https://doi.org/10.46954/revistages.v3i2.32

Giroux, H. (1992) Teoría de resistencia en la educación. Editores siglo XXI. México. Disponible en file:///C:/Users/azuce/Downloads/6855137_Henry_A_Giroux_Teoria_y_resisten.pd $\mathrm{f}$

Hernández Sampieri, R., Fernández Collado, C. y Baptista Lucio, P. (1991): Metodología de la investigación.

Jackson, P. (1970) La vida en las aulas. Madrid. Morata. Disponible en: https://www.academia.edu/24214241/

\section{LA_VIDA_EN_LAS_AULAS_PHILIP_JACKSON}

McGraw-Hill. Bernardo Carrasco, J. y Calderero, J. F. (2000): Aprendo a investigar en educación. Rialp. 2000.

Murillo Pacheco, H. (2010). CURRICULUM, PLANES Y PROGRAMAS DE ESTUDIOS. Obtenido de https://web.oas.org/childhood/ES/Lists/Recursos\%20\%20Estudios\%20e\%20Investi gaciones/Attachments/34/27.\%20Curri,\%20plan.pdf

Rivera, M. (2015) Influencia del currículo oculto en el desempeño académico, de los y las estudiantes del grado de 6.3 de la institución educativa Celmira Huertas. Colombia.

Tapia López, M. C. (octubre 2016 - enero 2017 de 2017). Evaluación del currículum real de la carrera Administración Turística y Hotelera. Torreón Universitario(Año 5 | No. 14 ). Obtenido de https://repositorio.unan.edu.ni/6292/2/172-467-1-SM.pdf

Taylor, P. (2008). EL CURRÍCULO DE LA EDUCACION SUPERIOR PARA EL DESARROLLO HUMANO Y SOCIAL. La Educación Superior en el mundo, 101.

Toro Santacruz, S. E. (2017). Conceptualización de currículo: su evolución histórica y su relación con las teorías y enfoques curriculares en la dinámica educativa. $a$ Publicando, 4 No 11. (1). 2017, 459-483. ISSN 1390-9304. 
Toro Santacruz, S. E. (2017). Conceptualización de currículo: su evolución histórica y su relación con las teorías y enfoques curriculares en la dinámica educativa. $a$ Publicando, 4 No 11. (1). 2017, 459-483. ISSN 1390-9304.

Vázquez Recio, R., López-Gil, M. y Calvo-García, G. (2019). El currículum oculto del fracaso escolar y del abandono educativo temprano. Razones interseccionadas. Investigación en la Escuela, 98, 16-30. Disponible en: http://dx.doi.org/10.12795/IE.2019.198.02 